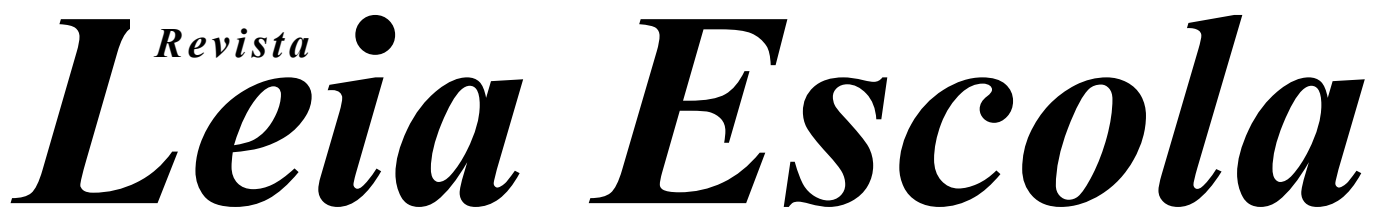

Programa de Pós-Graduação em Linguagem e Ensino 
UNIVERSIDADE FEDERAL DE CAMPINA GRANDE

Reitor Vicemário Simões

Vice-reitor Camilo Allyson Simões de Farias

Diretora do Centro de Humanidades Fernanda de Lourdes Almeida Leal

UNIDADE ACADÊMICA DE LETRAS

Coordenadora Administrativa Márcia Candeia Rodrigues

Coordenadores de Graduação

Licenciatura em Letras Espanhol

Licenciatura em Letras Francês

Licenciatura em Letras Inglês

Licenciatura em Letras Libras

Licenciatura em Letras Português

Coordenadora do Programa de Pós-Graduação

em Linguagem e Ensino

Coordenadora de Pesquisa e Extensão

Isis Milreu

Carmen Verônica A. R. Nóbrega

Cleydstone Chaves dos Santos

Aline Risseli Florindo Silva

Edmilson Luiz Rafael

Manassés Morais Xavier

Denise Lino de Araújo

Neide de Fátima Cesar da Cruz

Editora da UFCG Diretor Administrativo

José Hélder Pinheiro Alves

\section{Revista Leia Escola ISSN 2358-5870 \\ Editora Geral Márcia Tavares}

Comissão Editorial Denise Lino de Araújo

Quadriênio 2018-2021 Maria Augusta G. de Macedo Reinaldo

Naelza de Araújo Wanderley

Conselho Editorial

Aldinida Medeiros (UEPB) Lílian de Oliveira Rodrigues (UERN) André Pinheiro (UFPI) Luiz Francisco Dias (UFMG)

Angela Paiva Dionisio (UFPE) Maria Amélia Dalvi (UFES)

Amarino Queiroz (UFRN) Marco Antônio Margarido Costa (UFCG)

Ana Cristina Marinho Lúcio (UFPB) Maria Angélica de Oliveira (UFCG)

Ana Virgínia Lima (UFRN) Maria Auxiliadora Bezerra (UFCG)

Bruno Alves Pereira (UEPB) Maria Hozanete Alves de Lima (UFRN)

Darcília Simões (UERJ) Maria Marta dos Santos Silva Nóbrega (UFCG)

Daniela Segabinazi (UFPB) Marie Hélène Torres (UFSC)

Derivaldo dos Santos (UFRN) Renata Junqueira de Souza (UNESP)

Edenize Ponzo Peres (UFES) Roberto Carlos de Assis (UFPB)

Edmilson Luiz Rafael (UFCG) Sílvia Pilegi Rodrigues (UFMT)

Eliana Kefalas Oliveira (UFAL) Simone Dália de Gusmão Aranha (UEPB)

Fabiana Oliveira (UFAL) Sinara de Oliveira Branco (UFCG)

Fabiele Stockmans de Nardi (UFPE) Valdenides Cabral Dias (UFRN)

Fabiano Tadeu Grazioli (URI/FAE)

Fábio Marques de Souza (UEPB)

Fernanda Aquino Sylvestre (UFU)

Givaldo Melo de Santana (UFSE)

Josilene Pinheiro Mariz (UFCG)

José Hélder Pinheiro Alves (UFCG)

José Veranildo Lopes da Costa Junior (UERN)

Karina Chianca (UFPB)

Capa Leila Leite Santanta - UERN

Os trabalhos publicados são de responsabilidade exclusiva dos seus autores $\quad$ ISSN 2358-5870

Leia Escola - Revista do Programa de Pós-Graduação em Linguagem e Ensino da UFCG.

v. 20, n. 2, 2020. Campina Grande: EDUFCG, 2020.

1. Linguística

2. Linguística Aplicada

3. Literatura

4. Ensino 
UNIVERSIDADE FEDERAL DE CAMPINA GRANDE CENTRO DE HUMANIDADES

UNIDADE ACADÊMICA DE LETRAS
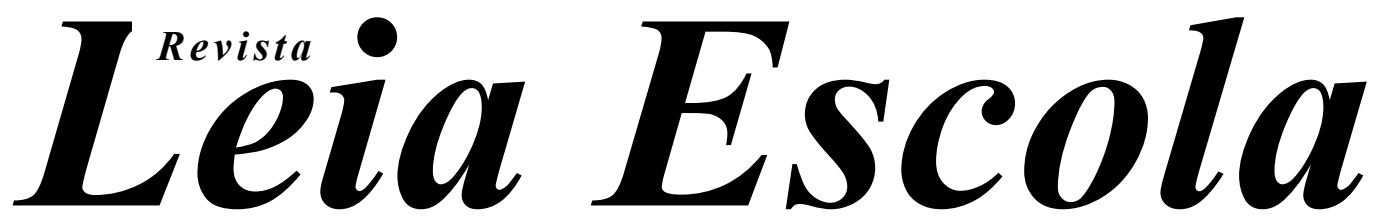

Programa de Pós-Graduação em Linguagem e Ensino

\title{
FORMAÇÃO DE PROFESSORES E TECNOLOGIAS DIGITAIS
}

\author{
Organizadoras \\ Dora Riestra - Universidade Nacional do Rio Negro - Argentina \\ Eulália Vera Lúcia Fraga Leurquin - UFC \\ Williany Miranda Silva - UFCG
}

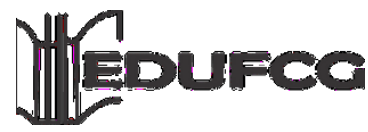

Campina Grande

2020 


\section{SUMÁRIO}

APRESENTAÇÃO.

DOSSIÊ TEMÁTICO

UM ESTUDO SOBRE O PERFIL TECNOPEDAGÓGICO DO PROFESSOR DE LÍNGUAS DO SÉCULO XXI: VOCÊ É TECNOFÓBICO, TECNOFÍLICO OU TECNOSTÊNICO?

Fábio Rodrigo Bezerra de Lima

A FORMAÇÃO INICIAL DO PROFESSOR DE LETRAS (E SUAS HABILITAÇÕES) E O USO DA TECNOLOGIA EM NOVOS GÊNEROS TEXTUAIS

Luciane Marlova Fontanela Puehler, Alice Atsuko Matsuda

A ABORDAGEM MULTIMODAL: UMA PROPOSTA DIDÁTICO-PEDAGÓGICA DE LETRAMENTOS DIGITAL E CRÍTICO

Mitia Risi dos Santos Costa

PROFESSORES NA ERA DIGITAL: ELABORAÇÃO DE UM CURSO DE FORMAÇÃO CONTINUADA SOBRE APLICATIVOS MÓVEIS

Kadhiny Mendonça de Souza Policarpo, Gabriela Marçal Nunes, Juliana Cristina Faggion Bergmann Correio.

NAVEGAR É PRECISO... LETRAR, TAMBÉM!: LETRAMENTOS DIGITAIS, PRÁTICAS EDUCACIONAIS E DISCUSSÕES EMERGENTES

Jennifer da Silva Gramiani Celeste, Juliana Célia de Oliveira.

A FERRAMENTA GOOGLE FORMS EM AVALIAÇÕES FORMATIVAS: A EFICÁCIA DE TECNOLOGIAS DIGITAIS NO ENSINO FUNDAMENTAL

Jacinta Antonia Duarte Ribeiro Rodrigues, Simone Dália de Gusmão Aranha, Fabiana Martins de Freitas.

APLiCATIVO ASSISTIVO COMO AUXILIADOR DA PRODUÇÃO TEXTUAL DE ALUNOS COM TEA

Vilma Mussilene de Araújo Candido

TECNOLOGIA COMO FERRAMENTA PEDAGÓGICA PARA O ENSINOAPRENDIZAGEM DA ORTOGRAFIA: ESTUDO COMPARATIVO ENTRE DOIS APLICATIVOS

Vera Aparecida Cordeiro Siqueira, Adriana Nascimento Bodolay, Luciana Pereira Assis

\section{ARTIGOS}

POESIA, RESISTÊNCIA E ESPERANÇA EM CONCEIÇÃO EVARISTO

Marcelo Medeiros da Silva, Josivânia da Cruz Vilela.

ENTREVISTA

ENSINO DE LÍNGUA E CULTURAS ESTRANGEIRAS EM TEMPOS DE (PÓS) PANDEMIA: DESAFIOS E APRENDIZAGENS DE EDUCADORES NA CHINA 